\title{
A 28 GHz 5G Phased Array Antenna with Air-Hole Slots for Beam Width Enhancement
}

\author{
Hojoo Lee $\mathbb{D}$, Sungpeel Kim and Jaehoon Choi *(D) \\ Department of Electronics and Computer Engineering, Hanyang University, Seoul 133-791, Korea; \\ hojoo31@hanyang.ac.kr (H.L.); spcl1@hanyang.ac.kr (S.K.) \\ * Correspondence: choijh@hanyang.ac.kr; Tel.: +82-2-2220-0376
}

Received: 9 September 2019; Accepted: 4 October 2019; Published: 9 October 2019

\section{Featured Application: 5G (28 GHz) mobile handset communication}

\begin{abstract}
In this paper, a $28 \mathrm{GHz}$ fifth-generation (5G) phased array antenna with air-hole slots for beam width enhancement is proposed. The proposed antenna consists of eight dipole radiators on a mobile handset-sized ground with air-hole slots between the two adjacent elements for enhancing the half power beam width (HPBW) in the elevation plane. The dimensions of the proposed antenna are $130 \mathrm{~mm} \times 42 \mathrm{~mm} \times 0.127 \mathrm{~mm}$. The proposed array antenna satisfies a $-10 \mathrm{~dB}$ reflection coefficient in the frequency range from 27.2 to $29.2 \mathrm{GHz}$ with a peak gain of $10.33 \mathrm{dBi}$ and a side lobe level (SLL) of $13 \mathrm{~dB}$. In addition to its good performance, the proposed antenna has a very wide HPBW (measured) in the elevation plane, up to 219 degree with a scan coverage of \pm 45 degree in the azimuth plane. The proposed antenna demonstrates excellent hemispheric beam coverage for $5 \mathrm{G}$ mobile handset devices and can enable cost-effective mass production.
\end{abstract}

Keywords: fifth-generation (5G); millimeter-wave; phased array; air hole; beam width

\section{Introduction}

With the rapid development of wireless mobile communication technologies, the demand for higher data rate services has significantly increased over the recent years. The fifth-generation $(5 \mathrm{G})$ mobile communication system [1] was introduced and has been receiving considerable interest from various industrial fields. Among many frequency bands, the millimeter band has been considered a good candidate for $5 \mathrm{G}$ communication, since it provides higher data rate services because of increased channel bandwidth compared to lower frequency bands [2]. A promising technology for utilizing millimeter band communications is the phased array antenna with beam steering capability [3]. Most studies have focused on the high peak gain, the wide reflection coefficient bandwidth, the wide scan angle, or the low side lobe level, which are typical specifications of the phased array antenna for mobile devices [4-6].

Very few, however, are interested in the half power beam width (HPBW) in the elevation plane [7-9], which is normal to the azimuth plane (beam scanning plane), as illustrated in Figure 1a. Since mobile communications require hemispheric beam coverage, as shown in Figure 1b [10], HPBW in the elevation plane (XZ plane) is a crucial specification of the phased array antenna needed to minimize the fading caused by the shaded area [11]. The antennas proposed in [7,8] employ substrate-integrated waveguide structure as a cavity and used posts in order to shape the beam pattern of the antenna. The HPBWs in the elevation plane of the antennas are 133.1 degree and 180 degree, respectively. The antenna proposed in [9] introduced parasitic loop and mushroom-like element in order to broaden the beam width up to 130 degree. However, such work either has high cost structure $[7,8]$ or beam width which is not wide enough for 5G application [9]. 


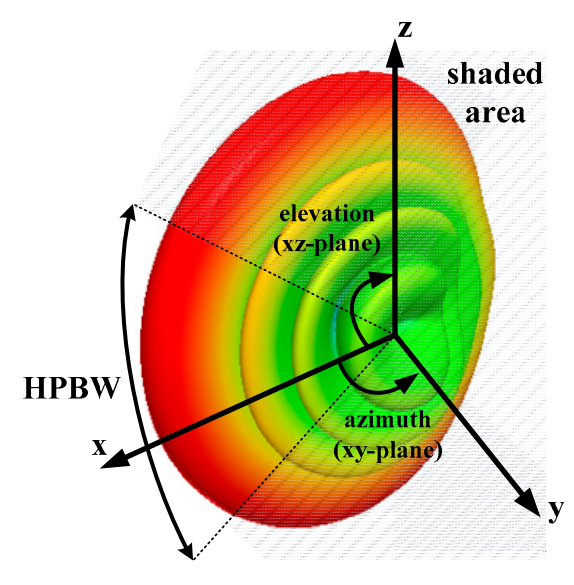

(a)

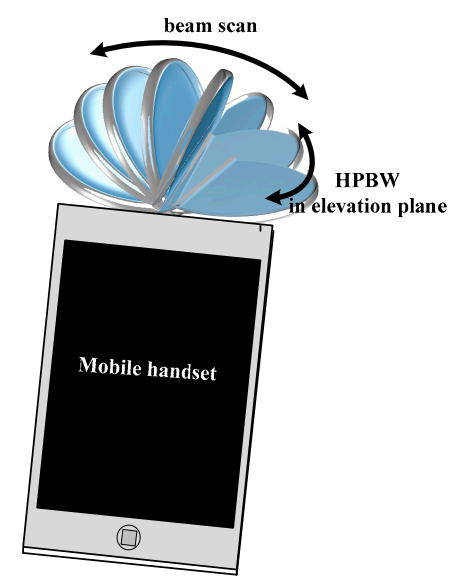

(b)

Figure 1. The azimuth and elevation plane versus the radiation pattern: (a) half power beam width (HPBW) in the elevation plane and shaded area; $(\mathbf{b})$ hemispheric beam coverage required by mobile handsets.

This paper proposes a $1 \times 8$ phased array antenna for $5 \mathrm{G}$ applications operating at $28 \mathrm{GHz}$ with enhanced HPBW in the elevation plane using air-hole slots. While showing adequate performances of the reflection coefficient bandwidth, the peak gain, the scan angle, and the side lobe level [12], the proposed antenna provides excellent HPBW in the elevation plane above 219 degree. The proposed array antenna has a relatively cost-effective structure compared with $[7,8]$ and has the widest HPBW (up to 39 degree) among the three studies [7-10]. Therefore, the proposed work can play an important role as array antenna for a modern $5 \mathrm{G}$ mobile handset device.

\section{Design of the Two-Element Antenna Array}

Figure 2a illustrates the structure of the proposed two-element antenna array. The proposed antenna consists of an air-hole slot in the ground substrate in addition to two bent dipole antenna elements and a modified ground. The two-element array antenna has dimensions of $10.7 \mathrm{~mm} \times 7.8 \mathrm{~mm} \times 0.127 \mathrm{~mm}$ and is printed on a Taconic RF-35 substrate with a relative permittivity of 3.5 and a loss tangent of 0.0018 .

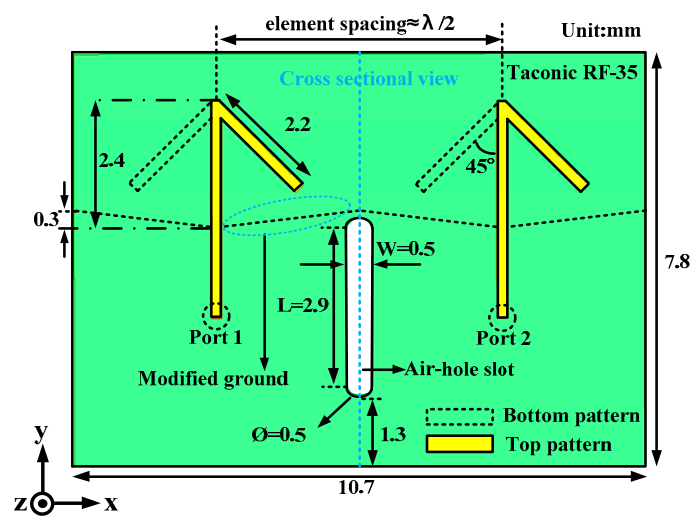

(a)

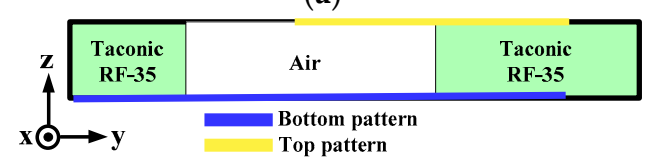

(b)

Figure 2. Structure of the proposed two-element array antenna: (a) top view (b) cross-sectional view. 
Bending of the dipole arms is applied to enhance the isolation between radiating elements and the front-to-back ratio (FBR) [13]. In addition, the ground plane is modified to improve the impedance matching of the antenna elements [14]. Figure 3 shows the reflection coefficient and isolation level of the proposed two-element array antenna. The proposed antenna exhibits a wide $(15 \%)-10 \mathrm{~dB}$ reflection coefficient bandwidth from $25.8 \mathrm{GHz}$ to $31 \mathrm{GHz}$ and an excellent isolation level higher than $21 \mathrm{~dB}$ over the desired frequency band. A slot (an air-hole slot) in the ground substrate is introduced to create the complimentary current component necessary to obtain the wide beam width. Figure $4 a, b$ shows the operating mechanism of the proposed ground slot enhancing the HPBW in the elevation plane of the two-element array. As shown in the dotted circle in Figure $4 \mathrm{a}$, the slot in the ground with length $\mathrm{L}$, approximately half of the guided wavelength, forms a complementary $\lambda / 2$-dipole originating via the current distribution around the slot, as described in the following equation [15]:

$$
L \simeq \lambda_{g} / 2
$$

where

$$
\lambda_{g}=\frac{\lambda_{0}}{\sqrt{\varepsilon_{r}}}
$$

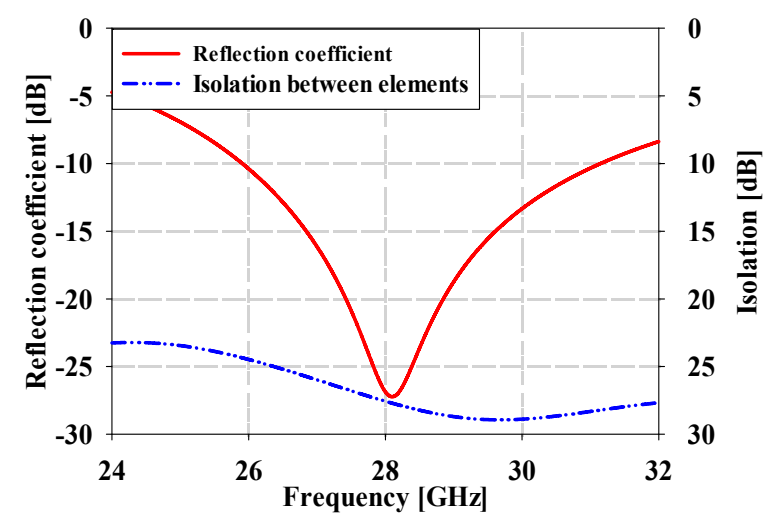

Figure 3. Simulated reflection coefficient and isolation level of the proposed two-element array.



(a)
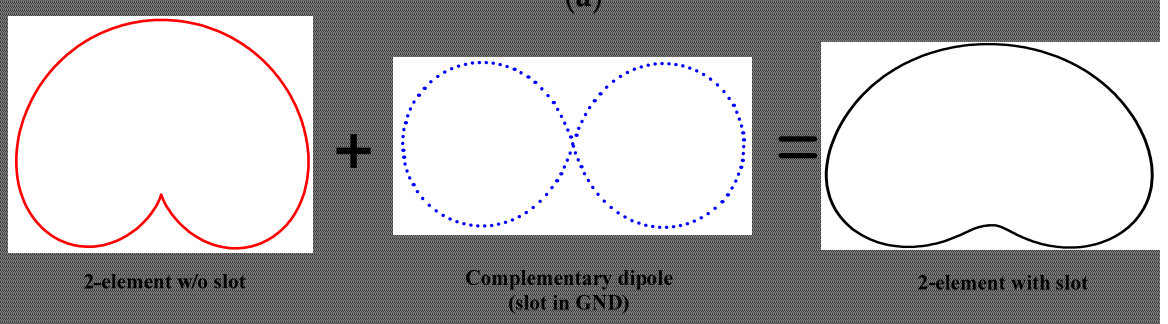

(b)

Figure 4. Implementation of the slot in the ground: (a) visualized mechanism of the slot as a complementary dipole source $(b)$ multiplication effect of the complementary dipole source on the radiation pattern of the two-element array. 
Therefore, the radiation pattern of the two-element dipole array is added to that of the complementary dipole from the ground slot resulting in a wider radiation pattern in the elevation plane, as shown in Figure $4 \mathrm{~b}[16,17]$. In addition, the width of the slot $\mathrm{W}$ is a key parameter for determining the performance of HPBW in the elevation plane.

Figure 5 illustrates the parametric study of the width $W$. The optimized value of $W$ is $0.5 \mathrm{~mm}$ since the peak gain starts to decrease at $\mathrm{W}=0.6 \mathrm{~mm}$ and the HPBW becomes extremely narrow at $\mathrm{W}=0.7 \mathrm{~mm}$, which implies that the slot does not operate properly as a complementary dipole radiator.

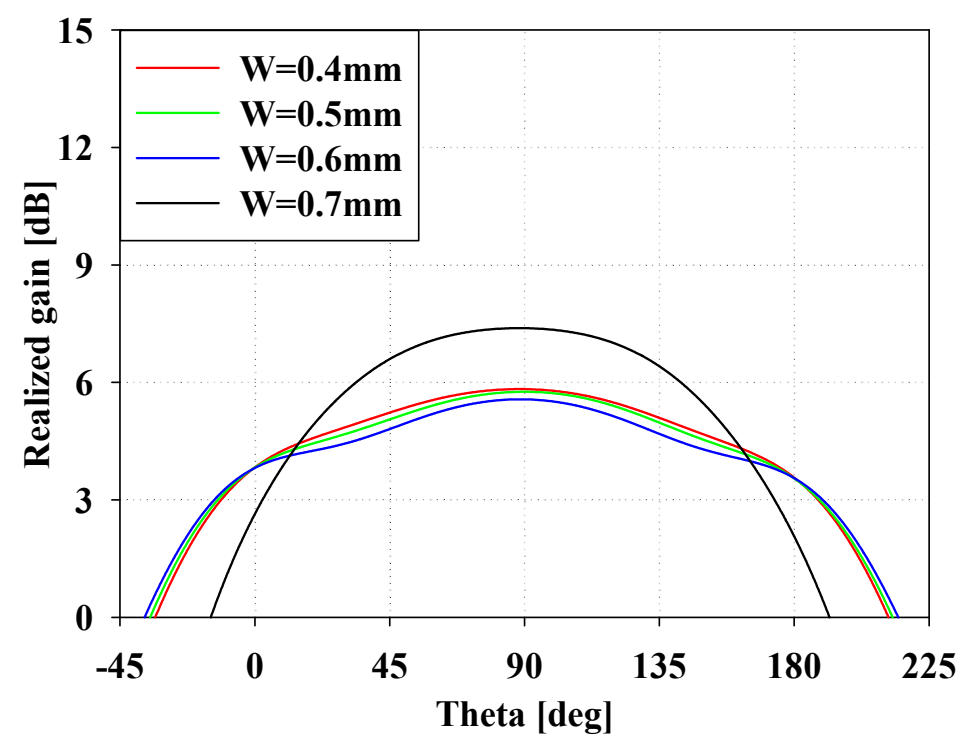

Figure 5. Realized gain for various values of the slot width (W).

To enhance the parasitic effect of the complementary dipole, an air-hole slot in the ground is introduced, as shown in Figure 2a. The air-hole slot consists of a slot on the ground and an air-hole made by puncturing the substrate. The air-hole has an identical location and size of the slot, and is laid on the top of the slot. The effect of such an air-hole slot can be simply explained by the complementary theory in which all the materials should be completely complemented for thorough implementation [18]. Such a theory can be further expanded upon using Maxwell's equations as following [19]:

$$
\begin{aligned}
\nabla \times H & =J+\varepsilon \frac{\partial E}{\partial t} \text { (Ampere's Law) } \\
\nabla \times E & =-u \frac{\partial H}{\partial t} \text { (Faraday's Law) }
\end{aligned}
$$

Qualitatively, the total surface current around the slot is increased since the focusing of E field caused by the difference of relative permittivity between air hole and the substrate reinforces the second term on the right hand side of the equation of Amperes' Law. This also increases the total magnetic field around the slot which is the left hand side of the Ampere's Law. Since the H field is time-varying, the right-hand side of the Faraday's Law increases and causally induces stronger total E field which is the left-hand side of the Faraday's Law. Eventually, the induced stronger E field around the slot enables the radiation of the complementary dipole to be enhanced. Because of the pattern summing mechanism illustrated in Figure $4 \mathrm{~b}$, the HPBW of the proposed array antenna in the elevation plane is improved. This is verified through observing the difference of the intensity of the surface current around the slot with and without the air hole. Figure $6 \mathrm{~b}$ shows the plot of the surface current ( $y$-axis) versus the distance around the slot ( $x$-axis) when it is applied alone and along with the air hole. 


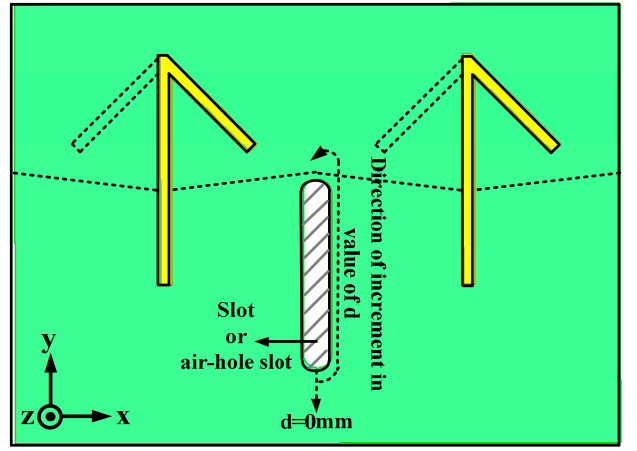

(a)

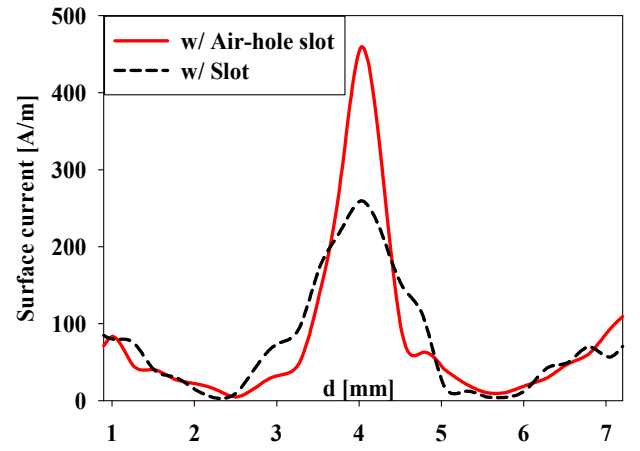

(b)

Figure 6. Introduction of the air-hole superposed with the slot in the ground: (a) top view of the simulation environment for surface current intensity around the slot (b) comparison of surface current intensity from the supposed two cases.

Figure 7 shows the radiation patterns of two-element arrays for three cases (i.e., without the slot, with the slot, and with the air-hole slot in the ground) in the YZ plane at $28 \mathrm{GHz}$. As we can see from the radiation pattern plot, the HPBW of the two-element array applied with the ground slot is increased from 190 degree to 203 degree compared with that without the ground slot. The HPBW is further increased to 207 degree in the $\mathrm{YZ}$ plane by introducing an air-hole slot instead of a ground slot.

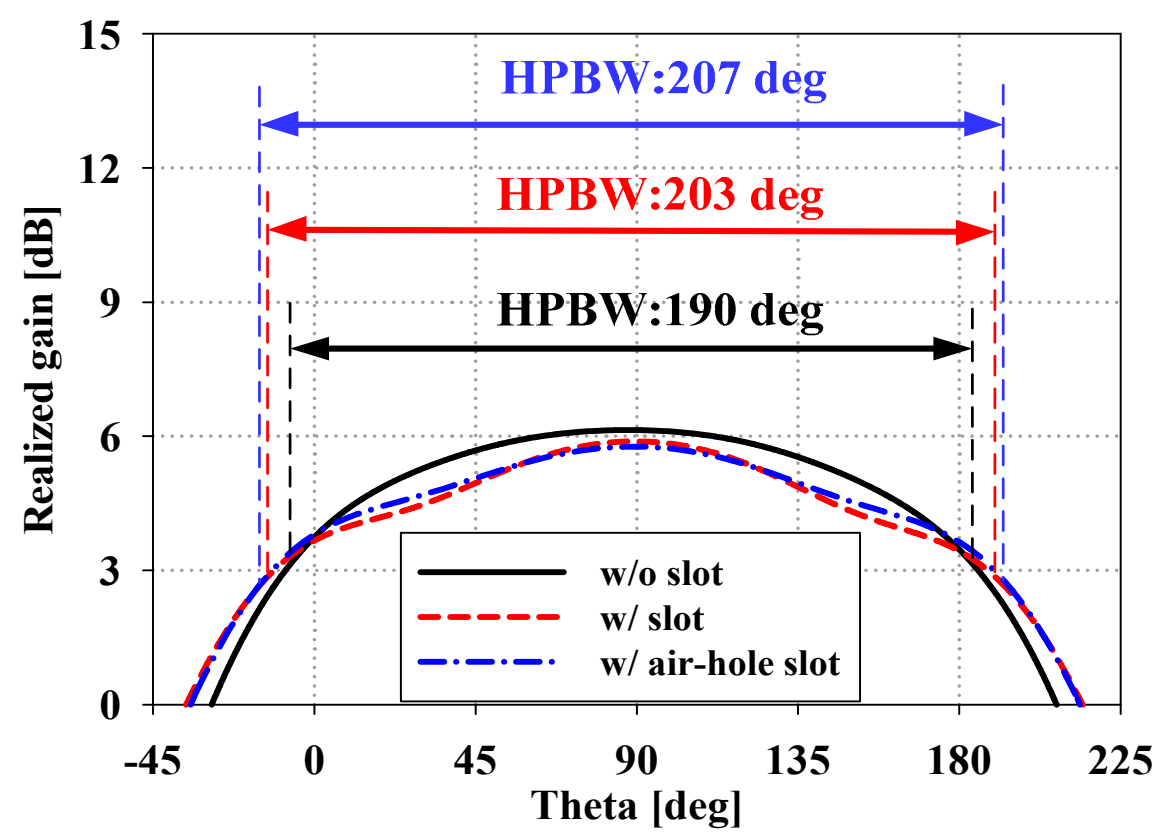

Figure 7. Comparison of HPBW at $28 \mathrm{GHz}$ in the $\mathrm{YZ}$ plane for the supposed three cases.

\section{Design of the Eight-Element Array on a Mobile Handset}

To verify the performance of a $5 \mathrm{G}$ phased array antenna on a typical mobile platform, the number of dipole elements is increased up to eight on a ground having the size of a typical mobile handset, as shown in Figure 8 . The $1 \times 8$ phased array antenna is located at the top side of the ground with the total dimensions of $130 \mathrm{~mm} \times 42 \mathrm{~mm} \times 0.127 \mathrm{~mm}$. The detailed antenna parameters and the substrate material are identical to those of the two-element array case. 


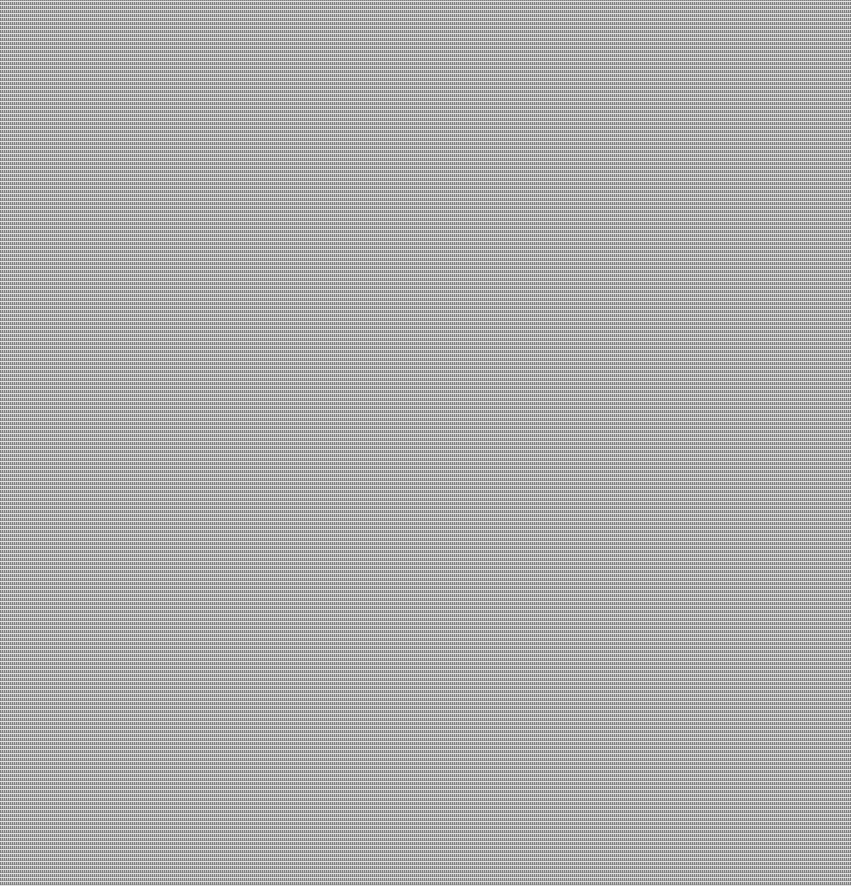

Figure 8 . Top view of the proposed $1 \times 8$ phased array antenna on a mobile handset ground.

As shown in Figure 9, the proposed antenna exhibits a $-10 \mathrm{~dB}$ reflection coefficient bandwidth of $5.1 \mathrm{GHz}$ while having the worst-case isolation level of $22 \mathrm{~dB}$ within that frequency bandwidth range. Figure 10a,b illustrate the radiation pattern of the proposed array antenna at $28 \mathrm{GHz}$ with a 0 degree phase difference between ports in the azimuth (XY plane) and elevation (YZ plane) planes, respectively. As shown in the figure, the peak gain in the azimuth plane is $11.15 \mathrm{dBi}$ toward +y direction with a side lobe level (SLL) of $13 \mathrm{~dB}$. On the other hand, the proposed antenna exhibits a very wide HPBW of 221 degree in the elevation plane with a peak gain of $11.15 \mathrm{dBi}$. Regarding such results, the air holes superposed with the slots in the ground can provide a wide HPBW to the array antenna in the elevation plane regardless of the number of elements. Figure 11 shows the beam-scanning performance of the proposed array antenna in the azimuth plane. As shown in the figure, the proposed antenna has a probable scan angle of \pm 45 degree (i.e., a total of 90 degree resulting from the symmetric structure) while maintaining the worst-case peak gain and SLL of $10 \mathrm{dBi}$ and $12 \mathrm{~dB}$, respectively.

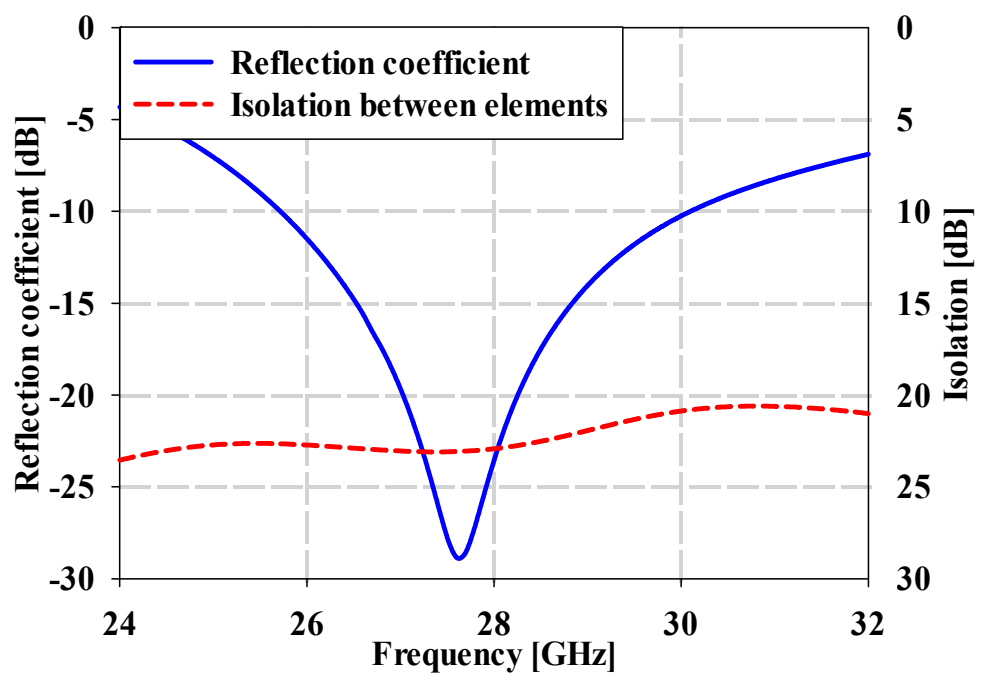

Figure 9. Reflection coefficient of the proposed antenna and isolation between the elements. 


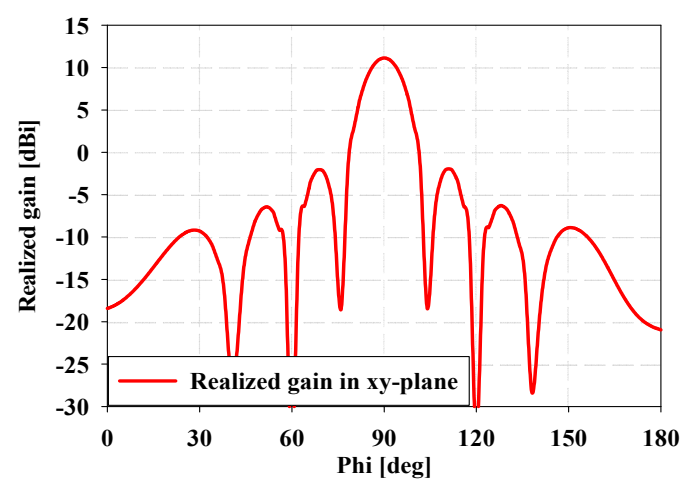

(a)

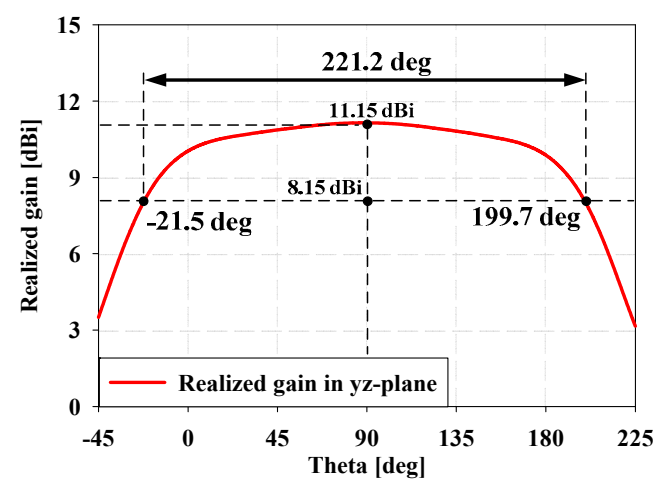

(b)

Figure 10. Radiation pattern of the proposed phased array antenna at $28 \mathrm{GHz}$ when the phase difference between element ports is 0 degree (0 degree scan): (a) XY plane (b) YZ plane.

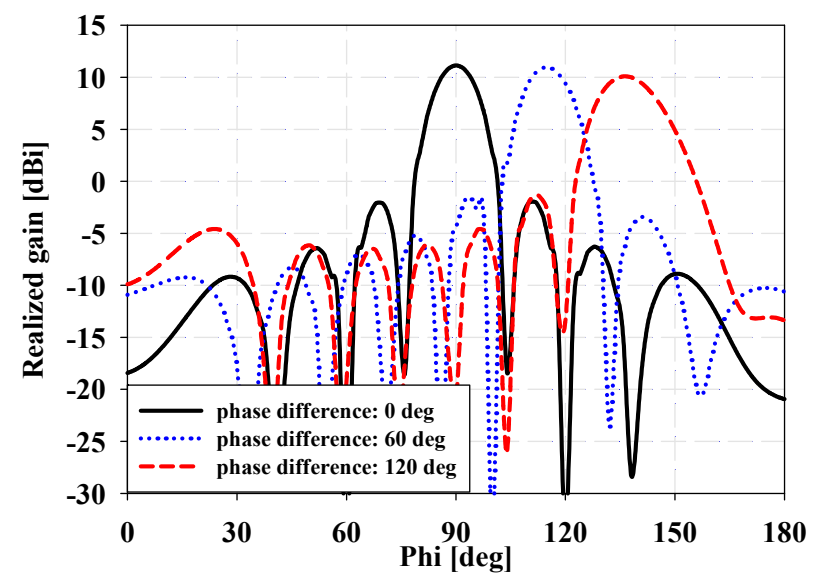

Figure 11. Radiation pattern of the proposed phased array antenna at $28 \mathrm{GHz}$ in the XY plane (azimuth) when the phase difference between element ports is varied from 0 degree to 120 degree with a step of 60 degree.

In summary, the proposed antenna exhibits adequate performances of reflection coefficient bandwidth, isolation level, peak gain, scan angle, and SLL [13] while having very wide HPBW, up to 221 degree in the elevation plane. Therefore, the proposed antenna can provide coverage of more than half of a hemisphere as a sub-array element, since it covers 90 degree in the azimuth plane with beam scanning and 221 degree HPBW in the elevation plane. If four proposed $1 \times 8$ phased array antennas are mounted on four corners of the mobile handset device, they can thoroughly cover the whole isotropic sphere without having a shaded area, as shown in Figure 1. Such characteristics can be a very crucial advantage when developing an array antenna system for 5G mobile handset applications.

\section{Experimental Results}

To verify the beam-steering performance, the proposed antenna was fabricated as a $1 \times 8$ mobile handset array antenna, as shown in Figure 12a. Etching process was applied to print the metal patterns on both sides of the substrate while simple drilling was used to realize the air-hole slot. Two types of fixed microstrip feeding networks were implemented to represent the linear phase differences of 0 degree and 120 degree for a 0 and 45 degree scan, respectively, as shown in Figure $12 b$. The substrate material and detailed antenna parameters for the proposed array antenna are identical to those of the two-element array design. 


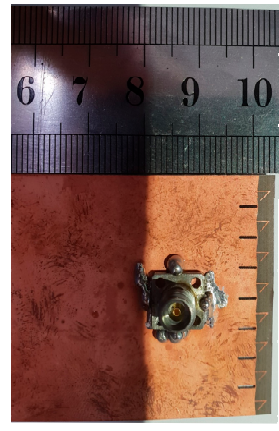

(a)

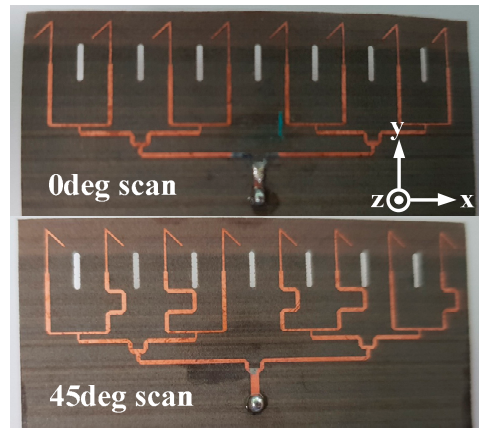

(b)

Figure 12. Fabricated $1 \times 8$ array antenna: (a) top view (b) bottom view

For measuring the reflection coefficient and the radiation pattern, Agilent N5230A vector network analyzer with Agilent/HP 85056D calibration kit and millimeter-wave anechoic chamber equipped with MTG operating software were used, respectively.

Figure 13 illustrates the measured reflection coefficients at the input port of the 18 array antenna with fixed microstrip feeding networks. The proposed array antenna has a 10-dB reflection coefficient bandwidth from 27.2 GHz to 29.2 GHz. As show in Figure 13, the measured results agree reasonably well with the simulated ones implemented with the fixed feeding network. A slight discrepancy between the simulated and measured results can be explained as an outcome of errors from measuring and manufacturing.

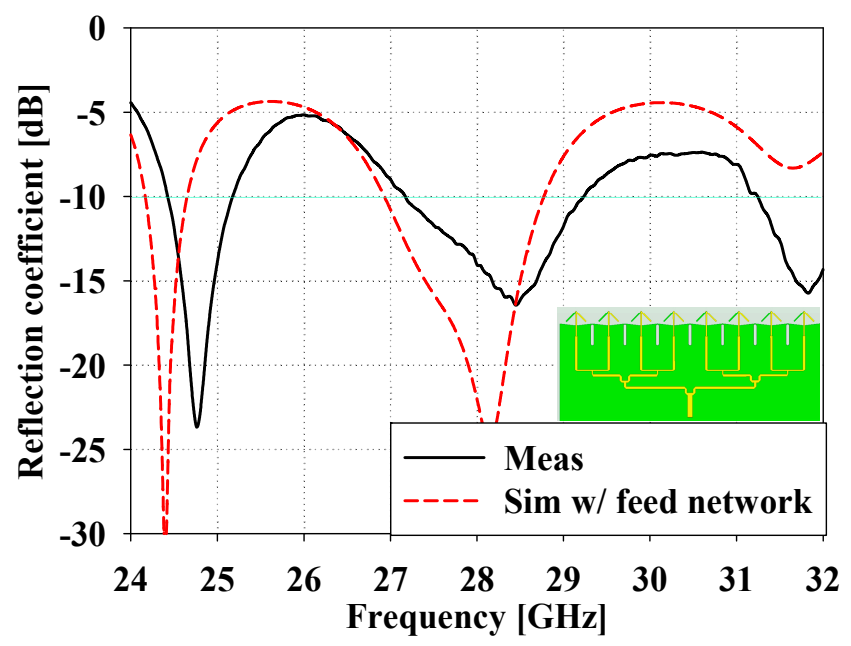

Figure 13. Measured and simulated reflection coefficient of the proposed array antenna.

Figure 14 shows the measured radiation patterns of the proposed array antenna at $28 \mathrm{GHz}$ along with the simulated results. Since the available anechoic chamber was technically specified to provide accurate results only for a rotational range from -120 to +120 degree relative to the boresight, plots in Figure 14 are illustrated within that range. As shown in Figure 14a,b, the measured peak gain of the array antenna with a 0 -degree scan is $10.33 \mathrm{dBi}$ in the $\mathrm{XY}$ plane with a HPBW of 219 degree in the $\mathrm{YZ}$ plane. As shown in Figure 14c, the measured peak gain of the array antenna with a 45 degree scan is $9.64 \mathrm{dBi}$ in the $\mathrm{XY}$ plane with an SLL of $10.1 \mathrm{~dB}$. As shown in the figures, the measured radiation patterns reasonably agree well with the simulated results, except for a small discrepancy in the gain value of approximately $0.8 \mathrm{~dB}$, which can be interpreted as an experimental error, including loss from the feeding connector. In summary, the fabricated antenna shows very wide HPBW in the elevation plane, while maintaining adequate performance in the other criteria, and agreeing reasonably well with the simulated results. 


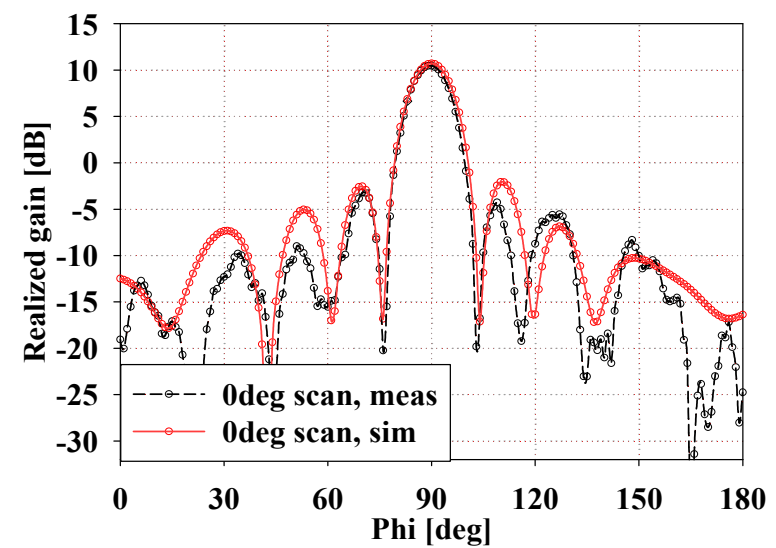

(a)

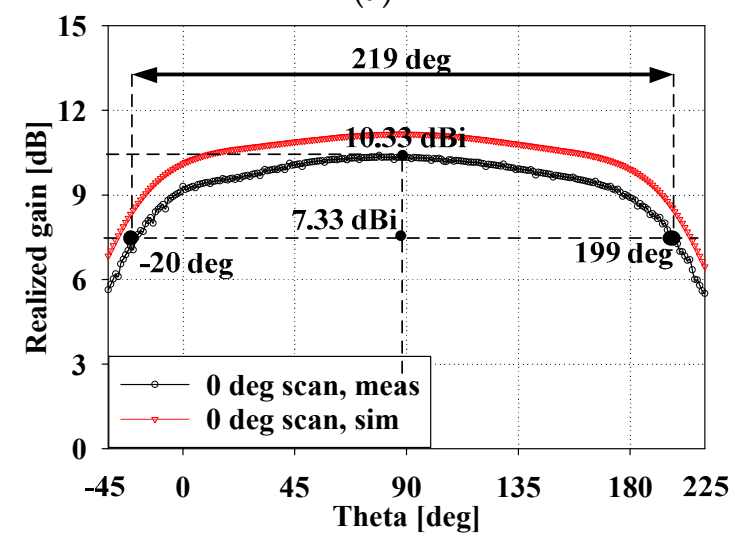

(b)

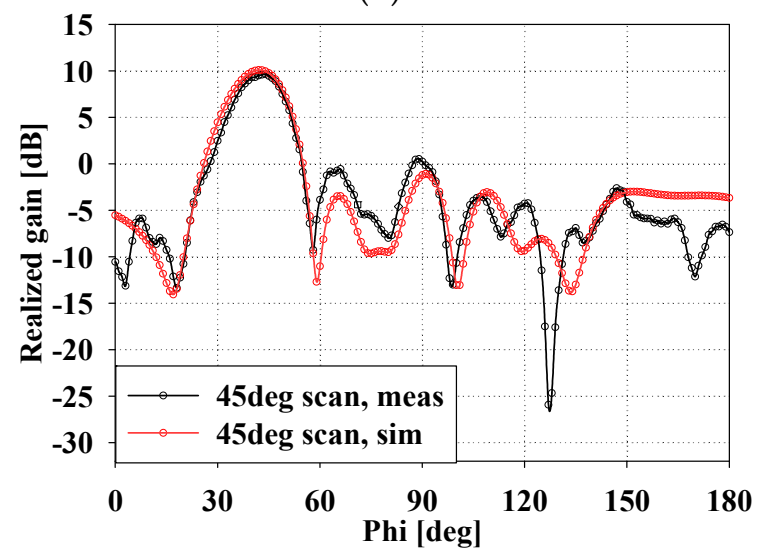

(c)

Figure 14. Measured and simulated radiation patterns of the proposed array antenna at $28 \mathrm{GHz}$ : (a) $X Y$ plane with a 0 degree phase difference; (b) YZ plane with a 0 degree phase difference; (c) XY plane with a 120 degree phase difference.

\section{Conclusions}

A $1 \times 8$ phased array antenna for $5 \mathrm{G}$ applications operating at $28 \mathrm{GHz}$ with enhanced HPBW is proposed in this paper. The proposed design is proven to have sufficient impedance matching, sufficient peak gain, a low sidelobe level, suitable scan coverage, and very wide HPBW in the elevation plane. The HPBW improvement over 20 degree is achieved by introducing air-hole slots, as compared to that without air-hole slots. Such enhancement of the HPBW in the elevation plane can be explained by the qualitative causality of two Maxwell's equations. Relatively good agreement between simulated and measured results can be explained by the simple structure of the proposed antenna since it has 
less factors for possible errors. Because of the very wide HPBW in the elevation plane along with reasonably good other radiation performances, the proposed array antenna can be a good candidate for the mm-wave 5G mobile handset applications. Future work will involve introduction of actual mobile handset model including metal chassis, circuit elements, and LCD panel. Since the performance of the antenna will be strongly affected by such inclusion, certain counter measures for minimizing the effect needs to be assessed. In addition, the effect of materials (dielectric or ferrite) that might be used to fill the air hole in actual situation, may also be taken into account.

Author Contributions: The presented work was conceptualized and written by H.L. and S.K., J.C. supervised the research.

Funding: This work was supported by Institute of Information \& Communications Technology Planning \& Evaluation (IITP) grant funded by the Korea government (MSIT) (2018-0-00732, Fundamental research for solving the bottleneck technology of millimeter wave $5 \mathrm{G}$ cellular array antenna).

Conflicts of Interest: The authors declare no conflict of interest.

\section{References}

1. Wells, J. Faster than fiber: The future of multi-G/s wireless. IEEE Microw. Mag. 2009, 10, 104-112. [CrossRef]

2. Kim, T.; Park, J.; Seol, J.; Jeong, S.; Cho, J.; Roh, W. Tens of Gbps support with mmWave beamforming systems for next generation communications. In Proceedings of the 2013 IEEE Global Communications Conference, Atlanta, GA, USA, 9-13 December 2013.

3. Roh, W. Millimeter-wave beamforming as an enabling technology for $5 \mathrm{G}$ cellular communications: Theoretical feasibility and prototype results. IEEE Commun. Mag. 2014, 52, 106-113. [CrossRef]

4. Yoon, J.; Yoon, Y. Bandwidth enhancement of single-layer microstrip reflectarrays with multi-dipole elements. J. Electromagn. Eng. Sci. 2019, 19, 130-139. [CrossRef]

5. Stanley, M.; Huang, Y.; Loh, T.; Xu, Q.; Wang, H.; Zhou, H. A high gain steerable millimeter-wave antenna array for 5G smartphone applications. In Proceedings of the 2017 11th European Conference on Antennas and Propagation, Paris, France, 19-24 March 2017.

6. Tekkouk, K.; Hirokawa, J.; Sauleau, R.; Ettorre, M.; Sano, M.; Ando, M. Dual-layer ridged waveguide slot array fed by a butler matrix with sidelobe control in the 60-GHz band. IEEE Trans. Antennas Propag. 2015, 63, 3857-3867. [CrossRef]

7. El-Halwagy, W.; Mirzavand, R.; Melzer, J.; Hossain, M.; Mousavi, P. Fence Shaping of substrate integrated fan-beam electric dipole for high-band 5G. Electronics 2019, 8, 545. [CrossRef]

8. Zhou, H.; Aryanfar, F. Millimeter-wave open ended SIW antenna with wide beam coverage. In Proceedings of the 2013 IEEE Antennas and Propagation Society International Symposium, Orlando, FL, USA, 7-13 July 2013.

9. Yu, C.; Li, E.; Jin, H.; Cao, Y.; Su, G.; Che, W.; Chin, K. 24 GHz horizontally polarized automotive antenna arrays with wide fan beam and high gain. IEEE Trans. Antennas Propag. 2019, 67, 892-904. [CrossRef]

10. Hong, W.; Baek, K.; Kim, Y.; Lee, Y.; Kim, B. mmWave phased-array with hemispheric coverage for 5th generation cellular handsets. In Proceedings of the 2014 8th European Conference on Antennas and Propagation, Hague, The Netherlands, 6-11 April 2014.

11. Andrews, J.G.; Bai, T.; Kulkarni, M.N.; Alkhateeb, A.; Gupta, A.K.; Heath, R.W. Modeling and analyzing millimeter wave cellular systems. IEEE Trans. Commun. 2017, 65, 403-430. [CrossRef]

12. Naqvi, A.H.; Lim, S. Review of recent phased arrays for millimeter-wave wireless communication. Sensors 2018, 18, 3194. [CrossRef] [PubMed]

13. Alhalabi, R.A.; Rebeiz, G.M. High-efficiency angled-dipole antennas for millimeter-wave phased array applications. IEEE Trans. Antennas Propag. 2008, 56, 3136-3142. [CrossRef]

14. Kim, S.; Han, J.; Jang, Y.; Choi, J. A compact VHF/UHF band quasi-Yagi antenna for multifunction radar applications. Microw. Opt. Technol. Lett. 2018, 60, 2525-2530.

15. Chen, W.K. The Electrical Engineering Handbook; Elsevier: Amsterdam, The Netherlands, 2005; p. 577.

16. Xiong, J.; Li, X.L.; Wang, B.Z. An idea for low-profile unidirectional slot antennas based on its complementary dipoles. In Proceedings of the 2013 International Symposium on Antennas and Propagation, Nanjing, China, 23-25 October 2013. 
17. Ye, M.; Li, X.; Chu, Q. Planar circularly polarized endfire antenna based on superposition of complementary dipoles. In Proceedings of the 2016 IEEE International Symposium on Antennas and Propagation, Fajardo, Puerto Rico, 26 June-1 July 2016.

18. Mushiake, Y. Self-Complementary Antenna; Springer Science and Business Media: Berlin, Germany, 2012; pp. 7-14.

19. Hayt, W.H.; Buck, J.A. Engineering Electromagnetics, 8th ed.; McGraw-Hill: New York, NY, USA, 2010; p. 208. 